\title{
Bem-estar subjetivo em mulheres participantes de um Programa Social na cidade de Sete Lagoas, Minas Gerais
}

\author{
Subjective well-being in women participating in a Social Program in city of Sete Lagoas, \\ Minas Gerais
}

Bienestar subjetivo en mujeres participantes de un Programa Social en la ciudad de Sete Lagoas, Minas Gerais

Cledilene Muniz de Oliveira ${ }^{1,2,5}$, Maria de Fatima de Matos Maia ${ }^{1,5}$ *, Celina Aparecida Gonçalves Lima $^{1,5}$, Jaime Tolentino Miranda Neto ${ }^{1,5}$, Berenilde Valéria de Oliveira Sousa ${ }^{1,5}$, Jean Claude Lafetá $^{1,5}$, Geraldo Magela Durães ${ }^{1,5}$, Italo Barreto Prates ${ }^{1,3,5}$, Ana Cristina Oliveira ${ }^{1,3,5}$, Thatiana Maia Tolentino ${ }^{4,5}$.

\section{RESUMO}

Objetivo: Analisar o bem-estar subjetivo e possível associação com as variáveis independentes consideradas em mulheres participantes de um programa social na cidade de Sete Lagoas, Minas Gerais. Método: Esta pesquisa possui caráter epidemiológico, transversal e analítico. A amostra contou com 298 mulheres praticantes de atividades orientadas. A coleta de dados foi feita por meio de questionário de avaliação modificado e ainda do questionário de Bem-Estar Subjetivo. Na análise dos dados, a variável bem-estar subjetivo foi considerada como variável dependente e as variáveis independentes foram a idade, o estado civil, a escolaridade, o índice de massa corporal, se possui filhos e bebida alcoólica. Utilizou-se a estatística descritiva por meio de frequências simples e relativas, além do teste $X^{2}$ para verificar a associação entre as variáveis independentes e o BES. Resultados: A maioria das mulheres tem idade de 40 anos ou mais $(65,2 \%)$, é casada/viúva $(59,6 \%)$ e é obesa $(54,3 \%)$. Apenas a variável escolaridade se mostrou associada ao bem-estar subjetivo. Conclusão: A maioria das mulheres participantes do programa social analisadas neste estudo possui o bem-estar subjetivo, os afetos positivos e as experiências positivas acima da média. $\mathrm{Na}$ análise do BES com as variáveis independentes, pode-se concluir que houve associação somente com a variável escolaridade.

Palavras-Chave: Programa Social, Mulheres, Bem-Estar.

\begin{abstract}
Objective: To analyze the subjective well-being and possible association with the independent variables considered in women participating in a social program in the city of Sete Lagoas, Minas Gerais Method: This research has an epidemiological, transversal and analytical character. The sample had 298 women practicing guided activities. The data collection was done through a modified evaluation questionnaire and the Subjective Well-Being questionnaire. In the data analysis, the variable SWB was considered as a dependent variable and
\end{abstract}

\footnotetext{
${ }^{1}$ Universidade Estadual de Montes Claros (UNIMONTES), Montes Claros-MG. ${ }^{2}$ Bolsista Iniciação Científica CNPq/UNIMONTES, Montes Claros-MG.

${ }^{3}$ Bolsista Iniciação Científica PROINIC FAPEMIG/UNIMONTES, Montes Claros-MG. ${ }^{4}$ Faculdade Santo Agostinho de Sete Lagoas (FASASETE), Sete Lagoas-MG.

${ }^{5}$ Grupo Integrado de Pesquisa em Psicologia do Esporte, Exercício e Saúde, Saúde Ocupacional e Mídia (GIPESOM). Montes Claros-MG. * E-mail: fatima.maia204@gmail.com
} 
the independent variables were age, marital status, schooling, body mass index, if have children and alcoholic beverage. Descriptive statistics were used by means of simple and relative frequencies, in addition to the $X^{2}$ test to verify the association between the independent variables and SWB. Results: The majority of women are 40 years of age or older (65.2\%), married/widowed (59.6\%) and obese (54.3\%). Only the variable education was associated with subjective well-being. Conclusion: Most of the women participating in the social program in this study have subjective well-being, positive affects and above-average positive experiences. When analyzing SWB with the independent variables, it can be concluded that there was an association only with the educational variable.

Keywords: Social Program, Women, Well-Being.

\section{RESUMEN}

Objetivo Analizar el bienestar subjetivo y posible asociación con las variables independientes consideradas en mujeres participantes de un programa social en la ciudad de Sete Lagoas, Minas Gerais.. Método: Esta investigación tiene carácter epidemiológico, transversal y analítico. La muestra contó con 298 mujeres practicantes de actividades orientadas. La recolección de datos fue realizada por medio de un cuestionario de evaluación modificado y del cuestionario de BS. En el análisis de los datos, la variable bienestar subjetivo fue considerada como variable dependiente y las variables independientes fueron la edad, el estado civil, la escolaridad, el índice de masa corporal, si posee hijos y bebida alcohólica. Se utilizó la estadística descriptiva por medio de frecuencias simples y relativas, además del test $x^{2}$ para verificar la asociación entre las variables independientes y el Bienestar Subjetivo. Resultados: La mayoría de las mujeres tienen una edad de 40 años o más $(65,2 \%)$, está casada/viuda $(59,6 \%)$ y es obesa $(54,3 \%)$. Sólo la variable escolaridad se mostró asociada al bienestar subjetivo. Conclusión: La mayoría de las mujeres participantes del programa social analizadas en este estudio tienen el bienestar subjetivo, los afectos positivos y las experiencias positivas por encima de la media. Al analizar la asociación del BS con las variables independientes, se puede concluir que hubo asociación solamente con la variable escolaridad.

Palabras Clave: Programa social, Mujeres, Bienestar.

\section{INTRODUÇÃO}

No decorrer da história da humanidade, a busca pela felicidade sempre esteve presente na vida das pessoas, por ser algo muito precioso, e desde a antiguidade foi um tema recorrente, pesquisado e discutido (DIENER SUH e LUCAS,1999). Na atualidade o estudo da felicidade é fator indispensável, porém com estrutura em valores diferenciados, resumida a atos de trabalho para adquirir dinheiro e para favorecer 0 consumismo e o modismo (SELIGMAN, 2004).

Entretanto, a busca por uma felicidade mais sólida tende a ter um referencial mais aprofundado, que envolve aspectos, como a realização pessoal, execução de um projeto de vida, a autoestima, o autoconhecimento, a participação em grupos de convivência, o convívio familiar, a saúde integral, a autonomia, a dignidade, a sexualidade, a espiritualidade e a paz (LUZ e AMATUZZI, 2008; PESSINI, 2014; PORTELLA et al., 2017). De acordo com Diener (2000) e Seligman (2004), o Bem-Estar Subjetivo (BES) é apontado como felicidade, podendo ser designado como extroversão estável, fazendo com que se entenda que os aspectos positivos na felicidade possam estar ligados a uma facilidade de se manter bons relacionamentos interpessoais. No entanto, quando o indivíduo não participa de algum grupo dentro da sociedade ou quando ele não possui um bom relacionamento interpessoal, a probabilidade de ele ser uma pessoa triste é maior.

Maia et al. (2016) afirmam que o conceito do bem-estar atual possui duas perspectivas científicas: o bemestar hedônico (acomete o estado subjetivo de felicidade) e o bem-estar eudemônico (acomete o bem-estar psicológico). De acordo com esses autores, são duas formas diferentes filosóficas de felicidade, pois a 
primeira (hedonismo) é tida como uma visão de bem-estar como prazer ou felicidade e a segunda (eudemonismo) dá a noção de que bem-estar consiste em sua capacidade de pensar, usar o raciocínio e o bom senso.

Corroborando com esses pensamentos, Myers e Diener (1995) e Portella et al. (2017) consideram que o bem-estar subjetivo, ao qual subjaz uma lógica hedônica de prazer e de gratificação mais imediatos, traduzse na presença de emoções positivas e ausência de emoções negativas e na avaliação global da satisfação com a vida. Para Portella et al. (2017), a maior satisfação com a vida parece estar relacionada com indicadores de funcionamento positivo, assim como menor satisfação com a vida tem sido associada à maior sintomatologia depressiva, à rejeição interpessoal e ao comportamento agressivo.

O BES vem sendo discutido na área acadêmica como uma nova visão dentro da área da psicologia e pode ser influenciado por variáveis como idade, gênero, nível socioeconômico e cultural (GIACOMONI, 2002). A literatura mostra que a atividade física possibilta melhora nas condições de vida, diminuindo as doenças e aumentando o bem-estar dos indivíduos, não contendo apenas uma dimensão biológica, pois o homem não deve ser avaliado apenas como um ser biológico, pois possui conteúdo histórico, social e psicológico (PESSINI 2014; PORTELLA et al., 2017).

Existem três domínios do bem-estar que se correlacionam com a avaliação global dos eventos, sendo Afeto Positivo e Satisfação de Vida de forma positiva e Afeto Negativo de maneira negativa, indo ao encontro da proposição de Diener et al. (1999) de que as interpretações subjetivas das circunstâncias objetivas são mais determinantes do BES do que as circunstâncias em si.

Neste estudo, ao utilizar o questionário Memorial University of Newfoundland Scale of Happiness (MUNSH), percebe-se que os afetos positivos, os afetos negativos, as experiências positivas e as experiências negativas passam a ser analisados como dimensões do bem-estar subjetivo, conforme preconiza Kozma e Stones (1980) em seu questionário.

Segundo Diener e Seligman (2004), os relacionamentos interpessoais e o estado civil das pessoas são correlatos do bem-estar subjetivo, pois os indivíduos não conseguem permanecer sozinhos, tendo a necessidade de estabelecer relações sociais positivas com outros indivíduos para o seu próprio bem-estar.

De acordo com Maia et al. (2016), existem fatores que podem interferir ou não no bem-estar subjetivo dos indivíduos. Estes podem ser: a idade, a escolaridade, o consumo de álcool, o Índice de Massa Corporal (IMC) e se possui filhos ou não, que passam a ser consideradas como variáveis independentes neste estudo.

Considerando o bem-estar do indivíduo e sabendo que o mesmo pode ser influenciado por aspectos sociais, históricos e econômicos, foi objetivo deste trabalho analisar o bem-estar subjetivo e possível associação com as variáveis independentes consideradas em mulheres participantes de um programa social na cidade de Sete Lagoas, Minas Gerais.

\section{MÉTODO}

Esta pesquisa possui caráter de estudo epidemiológico, transversal e analítico. A amostra foi composta por 298 mulheres praticantes de atividades orientadas nos polos (núcleos) de números 2 e 3, localizados na cidade de Sete Lagoas em Minas Gerais. Os dados dos núcleos foram fornecidos pela Prefeitura Municipal.

Os critérios de inclusão/exclusão foram assinar Termo de Assentimento Livre e Esclarecido (TALE) e o Termo de Consentimento Livre e Esclarecido em Pesquisa (TCLE) e estar frequente no dia da coleta. Outro critério é que se responda corretamente o questionário, não deixando questões em branco ou com respostas duplamente marcadas e estar fazendo atividade física nos dias da coleta.

Foram seguidos os procedimentos éticos com base na Resolução № 466, de 12 de Dezembro de 2012 da Comissão Nacional de Ética em Pesquisa (CONEP) do Conselho Nacional de Saúde (CNS). A pesquisa foi aprovada por um Comitê de Ética com Número do Parecer: 1.866 .770 com a data de 14 de Dezembro de 2016. 
A coleta de dados foi feita por meio de questionário de avaliação existente no programa modificado, já utilizado pela Secretaria de Esportes da cidade, e ainda do questionário de Bem-Estar Subjetivo MUNSH, de Kozma e Stones (1980), fornecido pelo Grupo Integrado de Pesquisa em Psicologia do Esporte, Exercício e Saúde, Saúde Ocupacional e Mídia (GIPESOM).

$\mathrm{Na}$ análise dos dados, a variável bem-estar subjetivo foi considerada como variável dependente e as variáveis: idade, estado civil, escolaridade, IMC, possui filhos e bebida alcoólica foram consideradas como variáveis independentes.

Utilizou-se a estatística descritiva, por meio de frequências simples e relativas, além do teste $X^{2}$ para verificar a associação entre as variáveis independentes e o BES. Foi adotado o nível de significância de $p \leq 0,05$.

Todas as análises estatísticas foram realizadas utilizando-se o programa Statistical Package for the Social Science (SPSS), versão 20.0.

\section{RESULTADOS}

Participaram dessa pesquisa 298 mulheres que eram participantes de um programa social, sendo a maioria $(64,8 \%)$ com idade de 40 anos ou mais e estado civil casada $(47,0 \%)$ (Tabela 1 ).

Tabela 1 - Caracterização das mulheres participantes do programa social.

\begin{tabular}{llcc}
\hline & VARIÁVEIS & $\mathbf{N}$ & $\%$ \\
\hline \multirow{2}{*}{ Idade } & Menos de 40 anos & 105 & 35,2 \\
& 40 anos ou mais & 193 & 64,8 \\
\hline \multirow{3}{*}{ Escolaridade } & Analfabeto/ensino fundamental & 103 & 34,8 \\
& Ensino médio & 147 & 49,7 \\
& Superior & 46 & 15,5 \\
\hline \multirow{3}{*}{ Estado Civil } & Solteira & 103 & 34,6 \\
& Casada & 140 & 47,0 \\
& Viúva/outro & 55 & 18,5 \\
\hline \multirow{2}{*}{ IMC } & Normal & 114 & 38,4 \\
& Sobrepeso & 105 & 35,4 \\
& Obesidade & 78 & 26,3 \\
\hline \multirow{2}{*}{ Possui Filhos } & Sim & 221 & 74,2 \\
& Não & 77 & 25,8 \\
\hline \multirow{2}{*}{ Bebida Alcoólica } & Sim & 97 & 33,4 \\
& Não & 193 & 66,6 \\
\hline
\end{tabular}

Fonte: Própria (2018).

Os resultados evidenciaram que a maioria das mulheres possuía um percentual maior de BES acima da média. Foram encontrados ainda percentuais acima da média para afetos positivos e experiências positivas (Tabela 2). 
Tabela 2 - Distribuição das dimensões do BES.

\begin{tabular}{llcc}
\hline \multicolumn{2}{c}{ DIMENSÕES DO BES } & N & $\%$ \\
\hline \multirow{2}{*}{ Afetos Positivos } & Abaixo da média & 130 & 44,4 \\
& Acima da média & 163 & 55,6 \\
\hline \multirow{2}{*}{ Afetos Negativos } & Abaixo da média & 187 & 63,0 \\
& Acima da média & 110 & 37,0 \\
\hline \multirow{2}{*}{ Experiências Positivas } & Abaixo da média & 134 & 45,1 \\
& Acima da média & 163 & 54,9 \\
\hline \multirow{2}{*}{ Experiências Negativas } & Abaixo da média & 154 & 51,9 \\
& Acima da média & 143 & 48,1 \\
\hline \multirow{2}{*}{ BES Geral } & Abaixo da média & 142 & 48,5 \\
& Acima da média & 151 & 51,5 \\
\hline
\end{tabular}

Fonte: Própria (2018).

Através da análise bivariada, a variável escolaridade das mulheres participantes do programa social mostrou-se associada ao BES (Tabela 3).

Tabela 3 - Análise bivariada do Bem-Estar Subjetivo (BES)

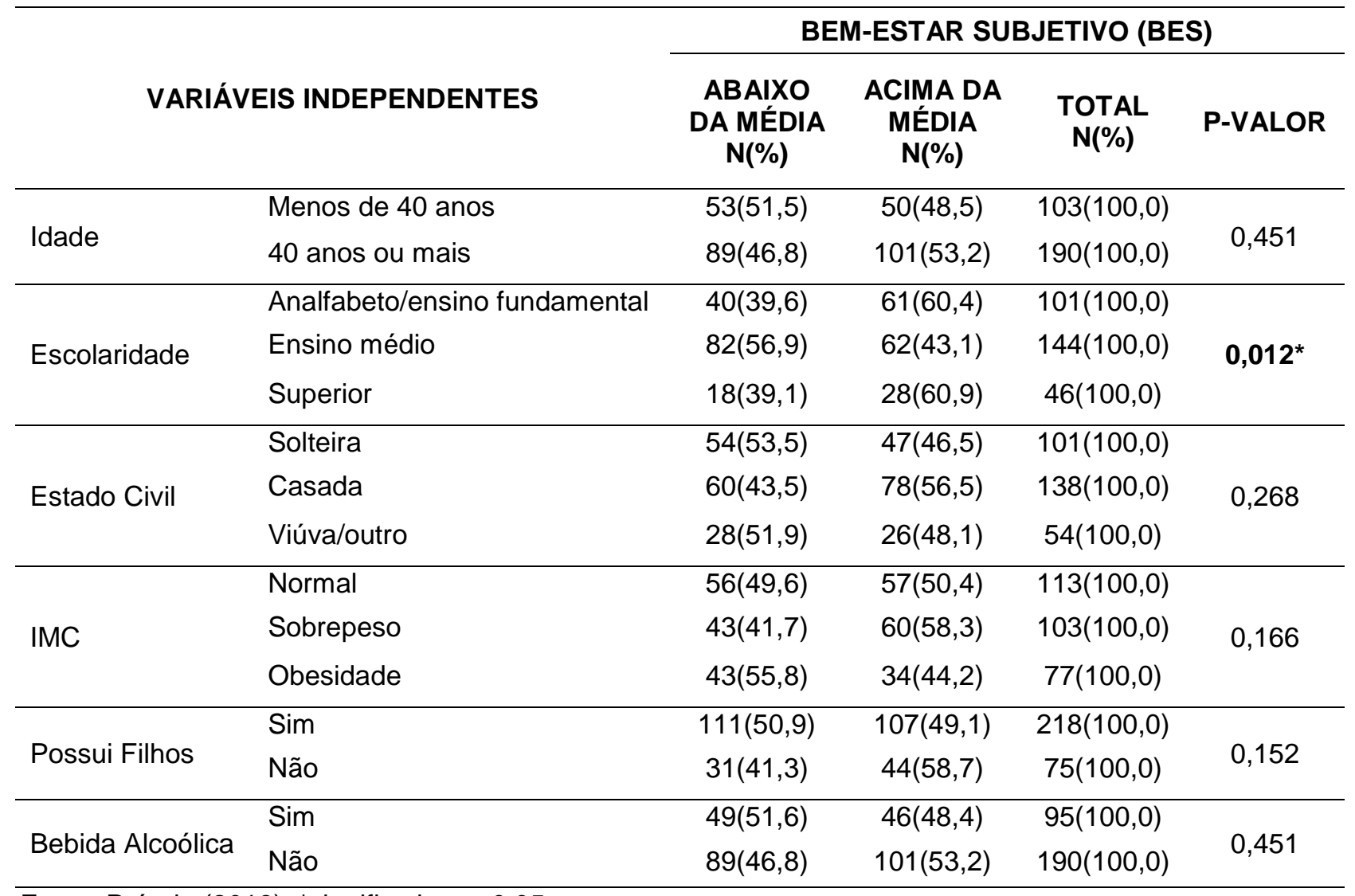

Fonte: Própria (2018); *significativo $p<0,05$. 


\section{DISCUSSÃO}

Este estudo analisou o bem-estar subjetivo de mulheres praticantes de atividade física orientada em um programa social. Das variáveis analisadas idade, escolaridade, estado civil, IMC, possui filhos e consumo de bebida alcoólica, apenas a variável escolaridade apresentou associação em relação ao BES. De acordo com a literatura, a escolaridade é apresentada como um dos principais determinantes do BES, com destaque direto para pessoas jovens e de meia-idade (GEORGE, 2010).

Estudo feito por Cachioni et al. (2017) com homens e mulheres idosas mostrou que o fato dos homens apresentarem maior escolaridade $(M=10.88)$ não refletiu no escore de satisfação geral com a vida e não houve evidência de resultados significativos dessa variável no presente estudo, corroborando com dados verificados por Yang (2008), que afirma também que a escolaridade está fortemente relacionada com a felicidade em todas as idades, exceto entre pessoas idosas.

No estudo de Queroz e Neri (2005), feito com pessoas adultas, notou-se que as variáveis idade e escolaridade mostraram resultados significativos, provando que adultos com alto nível de bem-estar psicológico e baixo nível de bem-estar subjetivo possuem um grau de escolaridade maior do que pessoas com um baixo nível de bem-estar psicológico e subjetivo, deixando em evidência que as combinações de bem-estar estão diretamente ligadas às variáveis idade e escolaridade.

De acordo com Cachioni et al. (2017), a educação está mais associada com a autopercepção da saúde do que com a avaliação de satisfação com a vida, pois tanto a escolaridade como as oportunidades para a educação em geral oportunizam atributos para que o indivíduo participe de atividades para aquisição de conhecimentos e mantenham relações sociais que estão ligadas ao BES, não sendo preditores de BES neste devido estudo.

Para Diener (1984), Diener et al. (1997) e Diener et al. (1999), a pessoa que possui alto bem-estar subjetivo está vivenciando satisfação de vida e emoções de contentamento e alegria. Portanto, emoções como tristeza e raiva são pouco presenciadas. O baixo bem-estar subjetivo é verificado quando a pessoa não está satisfeita com a sua vida e subsequentemente adquire emoções negativas. Seguindo a mesma ideia, Dela Coleta e Dela Coleta (2006) deixam esclarecida a opinião de que o bem-estar subjetivo se deve à satisfação de exigências relativas à saúde, à paz, às amizades, ao amor, ao dinheiro, à família, ao trabalho, às realizações, à religiosidade e à educação.

Ressalta-se a importância da escolaridade no BES de mulheres em decorrência da percepção das suas finalidades próprias quanto ao seu bem estar. Miranda Neto et al. (2012) colocam que a escolaridade, se efetiva como significativa nas experiências positivas sendo que as maiores médias vão para as pessoas quem possuem menor instrução. Os autores supracitados postulam que esse resultado pode advir da felicidade percebida em decorrência de poucas ambições e de estarem satisfeitos com sua vida.

A percepção que as pessoas obtêm sobre os eventos em sua vida, bem como suas maneiras de lidar com os mesmos, são de suma importância no estudo das relações entre os eventos de vida e o BES (CACHIONI, DELFINO, YASSUDA, 2017).

\section{CONCLUSÃO}

Conclui-se que a maioria das mulheres participantes de um programa social analisadas neste estudo possui o bem-estar subjetivo, os afetos positivos e as experiências positivas acima da média. Ao analisar a associação do bem-estar subjetivo com as variáveis independentes pode-se concluir que houve associação somente com a variável escolaridade. 


\section{REFERÊNCIAS}

1. CACHIONI M, DELFINO LL, YASSUDA MS et al. Bem-estar subjetivo e psicológico de idosos participantes de uma Universidade Aberta à Terceira Idade. Revista Brasileira de Geriatria e Gerontologia, 2017; 20(3): 340-352.

2. DELA COLETA JA, DELA COLETA MF. Felicidade, bem-estar subjetivo e comportamento acadêmico de estudantes universitários. Psicologia em estudo, 2006; 11(3): 533-539.

3. DIENER E. Subjective Well-Being. Psychological Bulletin. 1984; 95(3): 542-575.

4. DIENER E. Subjective well-being: The science of happiness and a proposal for a national index. American psychologist. 2000; 55(1): 34-43.

5. DIENER E, SELIGMAN MEP. Beyond Money: toward an economy of well-being. Psychological Science in the public interest. 2004; 5(1): 1-31.

6. DIENER E, SUH EM, LUCAS RE et al. Subjective well-being: Three decades of progress. Psychological bulletin. 1999; 125(2): 276-302.

7. DIENER E, SUH EM, OISHI S. Recent findings on subjective well-being. Indian journal of clinical psychology.1997; 24(1):25-41. 1997.

8. GEORGE LK. Still happy after all these years: research frontiers on subjective well-being in later life. J Gerontol Ser B Psychol Sci Soc Sci. 2010; 65(3): 331-339.

9. GIACOMONI CH. Bem-estar subjetivo infantil: conceito de felicidade e construção de instrumentos para avaliação. 2002; 1(1):1-250.

10. KOZMA A, STONES MJ. The measurement of happiness: Development of the Memorial University of Newfoundland Scale of Happiness (MUNSH). Journal of Gerontology. 1980; 35(6): 906-912

11. LUZ MMC, AMATUZZI MM. Experiences of happiness in elderly people. Estudos de Psicologia (Campinas). 2008; 25(2): 303-307.

12. MAIA MFM, RAPOSO JJBV, FORMIGA NS et al. Verificação empírica da consistência fatorial do inventário de bem-estar subjetivo MUNSH em jovens brasileiros. Psicologia em Pesquisa, UFJF. 2016; 10(2): 76-84.

13. MYERS DG, DIENER ED. Who is happy?. Psychological Science. 1995; 6(1):10-19.

14. MIRANDA NETO JT, LIMA CAG, GOMES MCS et al. Bem estar subjetivo em idosos praticantes de atividade física. Motricidade. 2012; 8(2): 1097-1104.

15. PESSINI L. Bioética, espiritualidade e a arte de cuidar em saúde. Envelhecimento humano, espiritualidade e cuidado. $2014 ; 2(1): 31-83$.

16. PORTELLA MR, SCORTEGAGNA HM, PICHLER NA et al. Felicidade e satisfação com a vida: voz de mulheres adultas e idosas. Revista Brasileira de Ciências do Envelhecimento Humano. 2017; 14(1): 93-101.

17. QUEROZ NC, NERI ALE. Bem-estar Psicológico e Inteligência Emocional entre Homens e Mulheres na Meiaidade e na Velhice. Psicologia: Reflexão e Crítica, 2005; 18(2): 292-299.

18. RORIZ P, PASCHOAL T. Relação entre ações de qualidade de vida no trabalho e bem estar laboral. Psicologia Argumento, Curitiba, 2012; 30(60): 585-593.

19. SELIGMAN MEP. Felicidade Autêntica: Usando a Nova Psicologia Positiva Para a Realização Permanente. 1 ed. Rio de Janeiro: Objetiva, 2004; 1(1): 1-266.

20. YANG Y. Social inequalities in happiness in the United States, 1972 to 2004: an age-period-cohort analysis. Am Socioll Rev. 2008; 73(2): 204-226. 